\title{
Extending the uranium-series dating of fossil coral reefs back to marine isotope stage 15
}

Claudine H. Stirling ${ }^{1}$ and Morten B. Andersen ${ }^{2}$

'Department of Chemistry, University of Otago, New Zealand; cstirling@chemistry.otago.ac.nz

2Department of Earth Sciences, University of Bristol, UK.

\begin{abstract}
Recent advances in U-series isotopic analysis allow absolutely dated records of past sea level change to be extended back through the last $600 \mathrm{ka}$, and offer decadal- to millennial-scale resolution for the last $400 \mathrm{ka}$.
\end{abstract}

The U-series dating of fossil coral reefs has been widely used to provide absolutely dated records of past sea level change and numerous observations now exist for the past 130 ka spanning the last glacial cycle (Edwards et al., 2003). To gain a more complete record of Earth's natural sea level cycles and a clearer understanding of the mechanisms driving climate change, it is important to study additional glacial-interglacial transitions further back in time. Extended, dateable records of past sea level change are also required to identify a suitable analog of the present climate system, in order to project future climate trends.

In general, the details of the sea level curve for the interval prior to $130 \mathrm{ka}$ remain elusive, primarily due to a progressive loss in the resolution of the U-series chronometer as one goes further back in time, coupled to a lack of well-preserved, dateable coral in older fossil reefs. The former issue is discussed in detail below. The latter is a major issue for corals of virtually all ages, due to post-depositional open-system disturbance of the U-series isotopic system leading to inaccurate ages (see Andersen et al., this issue). Efforts to ensure reliable ages involve strict isotopic screening criteria, often leading to the rejection of the majority of dated samples (Gallup et al., 1994) or the application of an open-system U-series model to correct for the altered component (Thompson et al., 2003; Thompson and Goldstein, 2005; Villemant and Feuillet, 2003; Scholz et al., 2004).

\section{Gearing up towards a high- precision chronometer}

The U-series chronometer uses the natural radioactive decay of ${ }^{238} \mathrm{U}$ to its longestlived intermediate daughters, ${ }^{234} U$ and ${ }^{230} \mathrm{Th}$. In general terms, the $\mathrm{U}$-series age of a sample can be determined from measurements of its ${ }^{234} \mathrm{U} / 238 \mathrm{U}$ and ${ }^{230} \mathrm{Th} /{ }^{238} \mathrm{U}$ compositions (Fig. 1A). The advent of multiplecollector thermal ionization (MC-TIMS), and more recently, inductively coupled plasma mass spectrometry (MC-ICPMS) to U-series dating in the 1980s and 1990s dramatically improved measurement precision compared with earlier techniques.

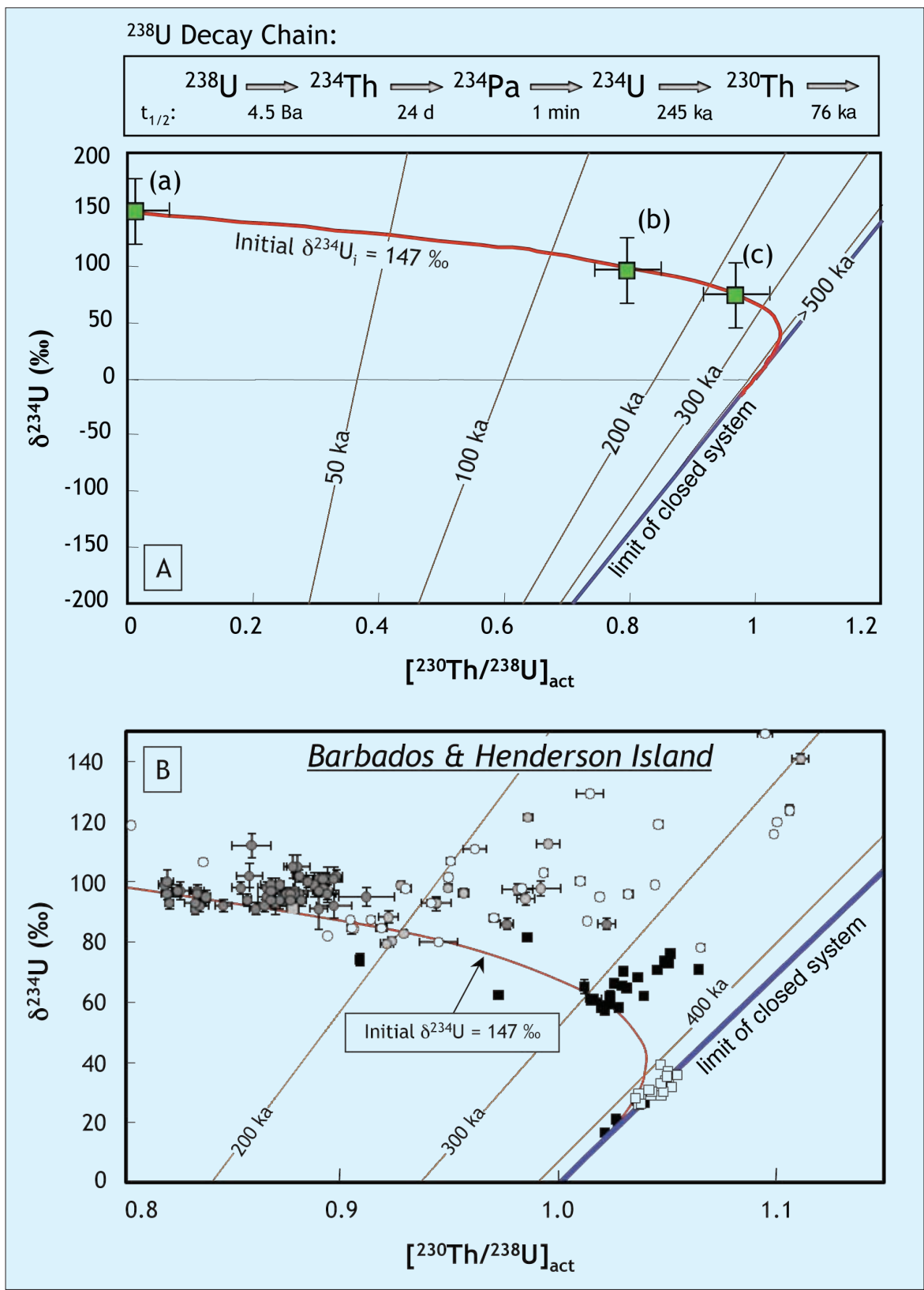

Figure 1: $A$ ) Typical representation of $U$-series observations for fossil coral reefs, whereby ${ }^{234} \mathrm{U} /{ }^{238} \mathrm{U}$ is plotted against ${ }^{230} \mathrm{Th} /{ }^{238} \mathrm{U}$ and contoured in units of $\mathrm{U}$-series age (near vertical lines). ${ }^{234} \mathrm{U} /{ }^{38} \mathrm{U}$ is reformulated into $\delta^{234} \mathrm{U}$-notation as the permil deviation away from radioactive equilibrium, and ${ }^{230} \mathrm{Th} / 238 \mathrm{U}$ is reformulated into the activity ratio $\left.{ }^{230} \mathrm{Th} /{ }^{38} \mathrm{U}\right] \quad$ (e.g. Edwards et al., 2003). For fossil corals, the U-series chronometer is based on the radioactive decay of ${ }^{234} \mathrm{U}$ (half-life $=245 \mathrm{ka}$ ) and radioactive in-growth of ${ }^{230} \mathrm{Th}$ ( $h$ alf-life $=76 \mathrm{ka}$ ) toward radioactive equilibrium with ${ }^{238} \mathrm{U}$. The initial $\delta^{234} \mathrm{U}=147 \%$ contour (red line) gives the closed-system evolution path for a coral, assuming a $\delta^{234} \mathrm{U}$ composition of $147 \% 0$ (identical to present-day seawater) and no ${ }^{230}$ Th at the time the coral formed, and no postdepositional loss/gain of ${ }^{238} \mathrm{U},{ }^{234} \mathrm{U}$ and ${ }^{230}$ Th other than by radioactive decay. A living coral would plot at position (a), whereas older $125 \mathrm{ka}$ and $225 \mathrm{ka}$ fossil corals would plot at positions (b) and (c) respectively, as shown by the three data points (green squares). The three data points (measured with identical levels of precision) also demonstrate that the resolution of the U-series chronometer decreases with increasing sample age as the separation between isochrons (orange lines) decreases. If the U-series system becomes open at any time, the isotopic composition of the coral will move away from the closed-system curve and will follow a new decay path, assuming the system remains closed afterward. B) Compilation of published U-series observations pre-dating the last glacial cycle from reef complexes in Barbados (circles) and Henderson Island (squares) displayed as $\delta^{234} \mathrm{U}$ versus [ [230Th/238 $\mathrm{U}$ ]. The initial $\delta^{234} \mathrm{U}=147 \%$ o contour denoting the closed-system evolution path for a coral is shown for reference (red line). The majority of data plot above this curve due to open-system exchange of the U-series isotopes during diagenetic alteration and therefore yield "unreliable" U-series ages. The error bars are 20. 


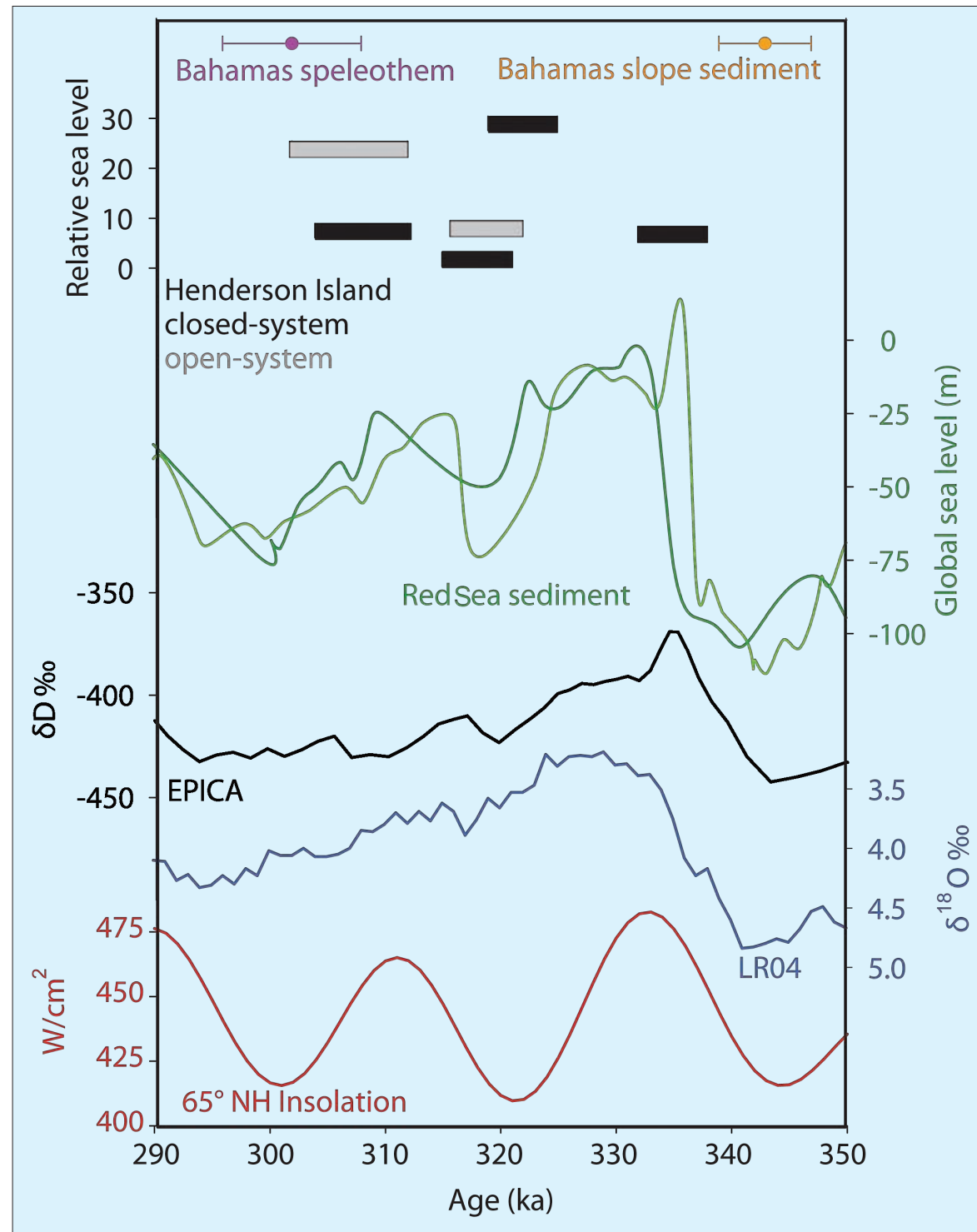

Figure 2: A compilation of sea level and climate records for MIS 9: Summer solar insolation predictions for latitude $65^{\circ} \mathrm{N}$ (red line), which according to the Milankovitch theory of climate change (Milankovitch, 1941), drive Quaternary glacial-interglacial climate variability; LR04 deep-sea sediment $\delta^{18} \mathrm{O}$ record (blue line; Lisiecki and Raymo, 2005), which comprises a stack of 57 globally distributed deep-sea $\delta^{18} O$ records, and provides a combined signal of deep-ocean temperature and global sea level for the past 5.3 Ma. The Pliocene-Pleistocene section of the record has been assigned a chronology based on orbital tuning to Milankovitch insolation predictions; $\delta D$ for the EPICA deep ice core from Dome C, Antarctica (black line; EPICA, 2004), which provides a proxy record of local air temperature for the past $740 \mathrm{ka}$. The chronology for the EPICA record has been derived using ice flow modeling, constrained by radiometric control points based upon Milankovitch orbital forcing theory; high-resolution global sea level reconstructions derived from the $\delta^{18} \mathrm{O}$ signatures of two independent Red Sea sediment cores (dark and light green lines; Siddall et al., 2003). The $\delta^{18} \mathrm{O}$ records were translated into global sea level using a complex hydraulic model for the exchange of water between the Red Sea and the open ocean. A chronology was assigned to the Red Sea records based on a combination of radiometric control points and synchronization with orbitally-tuned Antarctic ice core records; relative sea level observations based on the height-U-series age relationships of fossil corals sampled from Henderson Island's MIS 9 reef complexes but uncorrected for lithospheric flexural uplift and glacio-hydro-isostasy, are shown by the horizontal bars. Conventional U-series ages assessed as "reliable" on the basis of macroscopic and isotopic screening criteria are denoted by the black bars (Stirling et al., 2001); open-system U-series ages for other reef complexes that have been corrected for U-series isotopic shifts caused by diagenetic alteration using the open-system U-series model of Thompson et al. (2003) are represented by the gray bars; U-series observations for speleothems from the Bahamas (purple) constrain the timing of the MIS 8/9 glacial-interglacial transition (Hoffmann et al., 2007); Bahamian aragonitic slope sediments (orange) suggest an early onset for the initiation of MIS 9 (Henderson et al., 2006), which is contrary to the coral reef observations.

Using mass spectrometric techniques, ions generated in the thermal or plasma ionization source are passed through a magnetic field and spatially separated on the basis of their mass-to-charge ratio, then detected simultaneously on an array of Faraday detectors, devices that allow the precise measurement of ion currents. Isotope ratios are then determined on the basis of the ratio of the ion currents. Usually, Faraday detectors, although very stable, mentation over the last decade, conventional U-series measurement precision remains limited to the one-permil level because ion-counting detector systems are inherently unstable and must be frequently cross-calibrated (Goldstein and Stirling, 2003). When propagated through the $U$-series decay equations, one-permil uncertainties translate to sizeable U-series age errors in older samples of up to $\pm 5 \mathrm{ka}$ in 300 ka samples and $\pm 90 \mathrm{ka}$ in $600 \mathrm{ka}$ samples (Fig. 1). Clearly, age uncertainties of this magnitude are not conducive to sea level reconstruction at the millennial scale, nor do they allow the timing of an interglacial maximum to be resolved from the preceding glacial minimum for the interval prior to MIS 9 ( 350 ka).

New analytical protocols are required to improve the resolution of the U-series chronometer. To address this issue, some recent MC-ICPMS studies have focused on improving measurement precision by a further factor of five to the $0.2 \%$ level by adopting more concentrated solutions and increasing the minor ${ }^{234} \mathrm{U}$ and ${ }^{230} \mathrm{Th}$ ion beam signals to intensities that are sufficiently large for measurement on stable Faraday collectors (Andersen et al., 2004; 2007; 2008; Potter et al., 2005; Stirling et al., 2007; Cheng et al., 2008). MultipleFaraday protocols have also been applied to low-concentration thorium samples by overcoming the Faraday detector noise limitations (Esat, 1995; Stirling et al., 2001). There are several technical challenges that must be overcome to perform U-series isotopic measurements entirely using Faraday collectors. However, these challenges are offset by the fact that $0.2 \%$ levels of precision allow 300 and 600 ka samples to be measured with $2 \sigma$ age uncertainties of \pm 0.8 and \pm 14 ka, respectively. This represents a four-fold or better improvement in precision compared with previous techniques, and at the same time, extends the upper limit of the $\mathrm{U}$-series chronometer further back in time to $\sim 800 \mathrm{ka}$. Thus, multiple-Faraday techniques offer the potential to resolve the finer details of sea level and climate change during and beyond the last four glacial-interglacial cycles, especially when sample size is not restricted.

This enhanced analytical precision must be coupled with accuracy through continued refinement of the U-series half-life determinations to further reduce their contribution to the $\mathrm{U}$-series age error (Cheng et al., 2008). There is also a pressing need for all U-series laboratories to participate in a rigorous inter-laboratory comparison exercise, particularly given and ${ }^{238} \mathrm{U} /{ }^{230} \mathrm{Th} \sim 10^{5}$, it is therefore usual to measure the minor ${ }^{234} \mathrm{U}$ and ${ }^{230} \mathrm{Th}$ isotopes on a low-background ion counting detector system, a device for accommodating low-level ion currents. However, despite ongoing design improvements in instru- 
the rapid evolution of the $\mathrm{U}$-series field over the last decade.

\section{Sea level records pre-dating the last glacial cycle}

Prior to the last glacial cycle, the most extensive compilation of $\mathrm{U}$-series observations for fossil coral reefs exists for MIS 7 (Gallup et al., 1994; 2002; Scholz et al., 2006; Thompson and Goldstein, 2005; Dutton et al., this issue), extending from 245-193 ka. Sea level records for MIS 7 are discussed in detail by Dutton et al. (this issue). Furthermore there are a few isolated data points for MIS 6-5 (e.g., Esat et al., 1999; Thomas et al., 2008). Pre-dating MIS 7, the most extensive suite of coral reef U-series observations have been acquired for unusually well-preserved reef complexes formed during MIS 9 (339-303 ka) and MIS 15 (620-570 ka) on Henderson Island, South Pacific Ocean (Stirling et al., 2001; Andersen et al., 2008). Conventional U-series dating of Henderson Island's MIS 9 fossil corals (Fig. 2) indicate that sea levels during MIS 9.3 approached peak interglacial values near $324 \pm 3 \mathrm{ka}$ (Stirling et al., 2001). High sea levels appear to have persisted for a further $8 \mathrm{ka}$, before falling towards glacial values near $318 \pm 3 \mathrm{ka}$ (Stirling et al., 2001). Conventional U-series ages for a single coral tentatively indicate subsequent MIS 9.1 reef growth at $307 \pm$ 4 ka (Stirling et al., 2001). There are also a growing number of high-precision $\mathrm{U}$ series observations for Henderson Island's MIS 15 fossil corals and the results to date indicate peak interglacial sea levels at 600 \pm 15 ka (Andersen et al., 2008). Additional efforts are underway to continue to refine these MIS 9 and 15 sea level chronologies for Henderson Island (e.g., Stirling and Andersen, 2008). Furthermore, Henderson Island is believed to have undergone systematic but non-linear uplift ( 30 m) caused by lithospheric flexure arising from the volcanic emplacement of nearby Pitcairn Island. A combination of flexural uplift and glacio-hydro-isostatic rebound modeling is required to convert the relative sea level observations for Henderson Island into global constraints of eustatic sea level. To date, there is a complete absence of reliable $\mathrm{U}$-series observations for other glacial-interglacial periods. In particular, effort should be expended to identify MIS 11 in the fossil coral record. MIS 11 has been identified as an exceptionally warm and long interglacial in the climate record, characterized by orbital parameters that are similar to those of the today and of the next few millennia (EPICA, 2004), and could represent the most appropriate analog of the present climate system.

Efforts to pursue robust sea level records from fossil corals further back in time should focus on enhancing the precision and accuracy of the U-series chronometer.
Furthermore, an improved understanding of the mechanisms involved in the opensystem loss/gain of the U-series isotopes during the post-depositional diagenetic alteration of fossil reef systems (see Andersen et al., this issue) is critical to ensure the reliability of $\mathrm{U}$-series ages dated both conventionally and via the implementation of open-system methods. Moreover, it is important to augment the fossil coral records with U-series observations for complementary archives of past sea level change, particularly submerged speleothems (Bard et al., 2002; Dutton et al., this issue) and aragonitic slope sediments (Henderson et al., 2006).

\section{References}

Andersen, M.B., Stirling, C.H., Potter, E.K., Halliday, A.N., Blake, S.G., McCulloch, M.T., Ayling, B.F. and O'Leary, M., 2008: High-precision U-series measurements of more than 500,000 year old fossil corals, Earth and Planetary Science Letters, 265: 229-245.

Edwards, R.L., Cutler, K.B., Cheng, H. and Gallup, C.D., 2003: Geochemical Evidence for Quaternary Sea-level Changes. In: Turekian, K.K. and Holland, H.D. (Eds) Treatise on Geochemistry, Elsevier, 6.13 343-364.

Goldstein, S.J. and Stirling, C.H., 2003: Techniques for measuring uranium-series nuclides: 1992-2002. In: Bourdon, B. et al., (Eds) Reviews in Mineralogy and Geochemistry, Geochemical Society, 52: 23-57.

Stirling, C.H., Esat, T.M., Lambeck, K., McCulloch, M.T., Blake, S.G., Lee, D.-C. and Halliday, A.N., 2001: Orbital forcing of the Marine Isotope Stage 9 interglacial, Science, 291: 290-293.

Thompson, W.G. and Goldstein, S.L., 2005: Open-system coral ages reveal persistent suborbital sea-level cycles, Science, 308: 401404.

\section{Ice sheet retreat and sea level rise during the last deglaciation}

Peter U. Clark

Department of Geosciences, Oregon State University, Corvallis, USA; clarkp@onid.orst.edu

\section{The terrestrial record of deglaciation provides important constraints on the relative contribution of individual ice sheets to global sea level rise, thus improving our ability to estimate sea level sensitivity to climate change.}

The Last Glacial Maximum (LGM) is conventionally defined as the most recent interval in Earth's history when global ice sheets reached their maximum integrated volume. Documenting the rate of retreat of the global ice sheets following the LGM provides important insights towards understanding the sensitivity of ice sheet retreat and associated sea level rise to climate forcing. Additionally, a number of feedbacks associated with ice sheet retreat, including changes in albedo, orography, and freshwater fluxes to sites of deepwater formation, significantly influenced the trajectory and rate of deglaciation at regional to global scales.
The record of global sea level rise during the last deglaciation is best constrained from geomorphic and biological indicators of sea level at sites far from glaciated areas (far-field sites). Because sea level is an integrated signal, however, it does not distinguish between the relative contributions of individual ice sheets to the global signal, including growth of an ice sheet during the global deglaciation. Moreover, although the rate of sea level rise reveals changes in the total freshwater flux to the ocean associated with melting, it does not indicate how changes in the distribution of that flux to different regions of the ocean may have occurred solely from the rerouting of runoff, which may be just as important in influencing density-driven changes in ocean circulation as changes in the total flux.

Combining the terrestrial record of ice sheet retreat with the sea level record during the last deglaciation provides a complimentary strategy to address important aspects of ice sheet-climate interactions. Radiocarbon and cosmogenic surface exposure ages allow the position of retreating ice sheet margins to be mapped through the deglaciation, thus constraining rates of retreat (or readvance) for individual ice sheets as well as the opening (or closing) of outlets that route subcon- 